Int. J. Dev. Biol. 49: 17-21 (2004)

doi: $10.1387 / \mathrm{ijdb} .041906 \mathrm{kw}$

Original Article

\title{
Delivery of germinal granules and localized RNAs via the messenger transport organizer pathway to the vegetal cor- tex of Xenopus oocytes occurs through directional expan- sion of the mitochondrial cloud
}

\author{
KATARZYNA WILK ${ }^{1}$, SZCZEPAN BILINSKIㄴ, MATTHEW T. DOUGHERTY² and MALGORZATA KLOC*,3 \\ ${ }^{1}$ Institute of Zoology, J agiellonian University, Krakow, Poland, ${ }^{2}$ Biomedical Computation and Visualization Laboratory, Baylor College of \\ Medicine, National Center for Macromolecular Imaging, Houston, USA and ${ }^{3}$ Department of Molecular Genetics, The University of Texas, \\ M.D. Anderson Cancer Center, Houston, USA
}

\begin{abstract}
During Xenopus oogenesis, the message transport organizer (METRO) pathway delivers germinal granules and localized RNAs to the vegetal cortex of the oocyte via the mitochondrial cloud (Balbiani body). According to the traditional model, the mitochondrial cloud is thought to break up at the onset of vitellogenesis and the germinal granules and METRO-localized RNAs are transported within the mitochondrial cloud fragments to the vegetal cortex of the oocyte. We used light and electron microscopy in situ hybridization and three-dimensional reconstruction to show that germinal granules and METRO-localized RNAs are delivered to the oocyte cortex before the onset of mitochondrial cloud fragmentation and that the delivery involves accumulation of localized RNAs and aggregation of gemminal granules at the vegetal tip of the mitochondrial cloud and subsequent internal expansion of the mitochondrial cloud between its animal (nuclear) and vegetal tips, which drives the germinal granules and METRO-localized RNAs toward the vegetal cortex. Thus the fragmentation of the cloud that occurs later in oogenesis is irrelevant to the movement of METRO-localized RNAs and germinal granules. On the basis of these findings, we propose here a revised model of germinal granule and localized RNAs delivery to the oocyte vegetal cortex via the METRO pathway.
\end{abstract}

KEY WORDS: localized RNA, M ETRO pathway, mitochondrial cloud, Balbiani body, germinal granules

Introduction

The Balbiani body or mitochondrial cloud is a prominent cytoplasmic organelle that was discovered in 1845 in the oocytes of spiders and subsequently was found in the oocytes of many different species including humans (Guraya, 1979; de Smedt, 2000; Houston and King, 2000; Kloc et al., 2001, 2004a). The mitochondrial cloud present in previtellogenic stage I oocytes of Xenopus is composed of numerous mitochondria and germinal granules that are believed to be a determinant of germ cell fate (Eddy, 1975; Billet and Adam, 1976; Heasman et al., 1984). The cloud also contains various localized RNAs, some of which (e.g., Xcat2 mRNA) are present within the germinal granules and others (e.g., Xpat mRNA) are present at the periphery and outside the germinal granules (Mosquera et al., 1993; Kloc and Etkin, 1995a, b; Hudson and Woodland, 1998; Kloc et al., 2000, 2002, 2004b).
There are two main RNA localization pathways in Xenopus oocytes. The message transport organizer (METRO) or Early pathway uses the mitochondrial cloud as a vehicle to deliver germinal granules and localized RNAs to the oocyte vegetal cortex. In contrast, the Vg1 or Late pathway transports RNAs via microtubules without the participation of the mitochondrial cloud (Forristall et al., 1995; King, 1995; Kloc and Etkin, 1995a; Kloc et al., 1998). Conventional understanding holds that from early vitellogenesis (oocyte stage II) onward, the mitochondrial cloud breaks up into many fragments that become progressively smaller over time and migrate toward the vegetal cortex (Hausen and Ribesell, 1991). Upon their arrival at the vegetal cortex (between stages III and VI of oogenesis), fragments of the mitochondrial

A bbreviations used in this paper: METRO, message transport organizer;

\footnotetext{
*Address correspondence to: Malgorzata Kloc, Ph.D. Department of Molecular Genetics, The University of Texas, M.D. Anderson Cancer Center,1515 Holcombe BIvd. Houston, TX 77030, USA. Fax: +1-713-794-4394. e-mail: mkloc@mdanderson.org
} 
cloud (containing mitochondria, germinal granules and localized RNAs) become anchored at the cortex by actin and cytokeratin filaments; at this point they are called the germ plasm islands (Czolowska, 1969; Eddy, 1975; Kloc and Etkin, 1995a; Houston and King, 2000; Kloc et al., 2001, 2004a). Movement of the mitochondrial cloud fragments has been assumed to be responsible for delivering the localized RNAs and germinal granules embedded in those fragments to the oocyte vegetal cortex (reviewed in Kloc et al., 2004a, b). The movement of the mitochondrial cloud fragments toward the cortex is independent of microtubules and microfilaments and the mechanism that powers the movement remains a mystery (Kloc and Etkin, 1995a,b). The addition of newly synthesized yolk platelets and an increase in the cytoplasmic mass at the center of the oocyte have been suggested to push the fragments of mitochondrial cloud away from the oocyte nucleus and toward the vegetal cortex (Hausen and Riebesell, 1991; reviewed in Kloc et al., 2004a). Here we studied previtellogenic and early vitellogenic Xenopus oocytes by using a combination of three-dimensional reconstruction and in situ hybridization for light and electron microscopy and we showed that germinal granules and METRO-localized RNAs reach their ultimate destination before the onset of mitochondrial cloud fragmentation; thus we propose a revised model of germinal granule and localized RNAs delivery to the oocyte vegetal cortex via the METRO pathway.

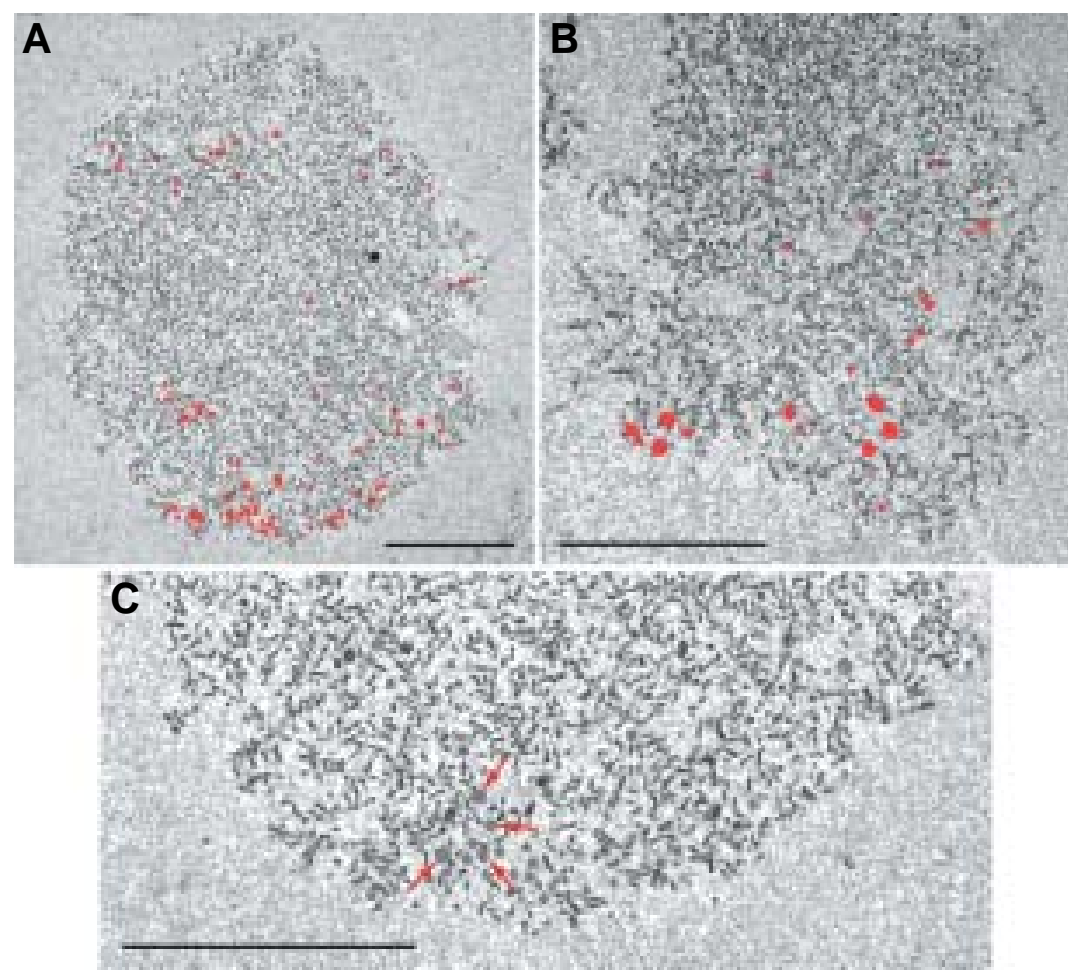

Fig. 1. Electron micrographs of cross/oblique sections of the mitochondrial cloud in Xenopus oocytes. (A) In stage I oocyte, the mitochondrial cloud is a spherical, compact organelle composed of thousands of mitochondria (gray specs) and hundreds of germinal granules. Germinal granules (in red) are mainly concentrated at the vegetal tip of the cloud. (B) Stage II oocyte shows many interconnected islands containing mitochondria (grey specs) and germinal granules (in red). Germinal granules were artificially colored. (C) METRO region of mitochondrial cloud from stage I oocyte showing germinal granules (red arrows) between mitochondria. Bars, $10 \mu \mathrm{m}$.

\section{Results and Discussion}

In Xenopus, the formation of the mitochondrial cloud and germinal granules is a lengthy and elaborate process that starts in oogonia and concludes in stage I oocytes (al-Mukhtar and Webb, 1971; Coggins, 1973; Billet and Adam, 1976;; Heasman et al., 1984; Wylie et al., 1985; Kloc et al. 2002, 2004a, b). In stage I oocytes, the mitochondrial cloud is a spherical organelle (approximately $40 \mu \mathrm{m}$ in diameter) that juxtaposes the nucleus and always faces the future vegetal pole of the oocyte. It contains about half a million mitochondria and several hundred germinal granules (Marinos and Billett, 1981; Kloc et al., 2002). Our previous three-dimensional reconstruction of the distribution of germinal granules within the mitochondrial cloud in stage I oocytes showed that most of the granules were located at the vegetal of the cloud in the METRO region. When the mitochondrial cloud was viewed from its vegetal tip, the granules were visible as a distinct ring and on a section passing exactly through the animalvegetal axis of the mitochondrial cloud, the granules were visible s a layer at the vegetal tip (Kloc et al., 2002). Until now, threedimensional reconstruction has never been performed on oocytes at later stages of development. However, incidental sections from light or electron microscopy suggest that beginning at stage II of oogenesis, the mitochondrial cloud breaks down into many fragments containing mitochondria and germinal granules (Hausen and Riebesell, 1991; Forristall et al., 1995; King, 1995; Kloc and Etkin, 1995a; Kloc etal., 1998, 2004a). The METRO region of the mitochondrial cloud contains several localized RNAs, such as Xcat2 mRNA (Mosquera et al., 1993) the distribution of which is restricted to the germinal granules and Xpat mRNA (Hudson and Woodland, 1998), which is present both at the granule periphery and outside the granules in the germ plasm matrix of the METRO region (Kloc et al. 2002). In this study, we performed light and electron microscopy in situ hybridization of early stage I, stage I, stage II and stage III Xenopus oocytes taking advantage of Xcat2 mRNA being a distinct marker of germinal granules. Electron microscopic sections of stage I oocytes showed that germinal granules were indeed concentrated at the vegetal tip of the mitochondrial cloud (Fig. 1 A,C). In sections of stage II oocytes granules were visible in the mitochondriacontaining islands at the vegetal tip of the oocyte (Fig. $1 \mathrm{~B})$, a finding that seems to support the conclusion that the mitochondrial cloud breaks down into smaller fragments at stage II of oogenesis. Our observation of the germinal granules in early stage I oocytes showed that germinal granules were more or less randomly distributed within the cloud (Fig. 2A). Subsequently all of the germinal granules aggregated at the vegetal tip of the cloud (Fig. 2B) and then formed a layer there (Fig. 2C), an arrangement that persisted during the later stages of oogenesis (Fig. 2 D,E). These findings indicate that the initial process of segregation and aggregation of germinal granules occured within the mitochondrial cloud itself. Solitary, evidently "delayed" germinal granules were occasionally visible at the animal (nuclear) tip of the cloud 


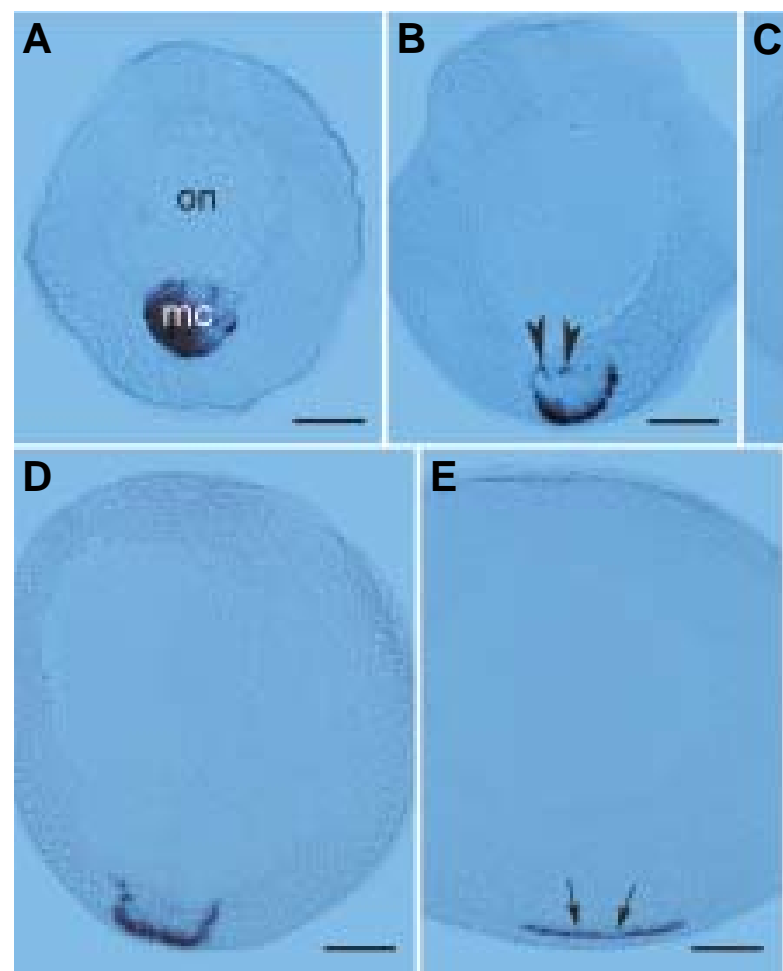
randomly within the whole cloud. (B) In early stage II oocyte the mitochondrial cloud extends between the nucleus and vegetal cortex and germinal granules are concentrated as a layer at its vegetal tip (the METRO region). Several "delayed" germinal granules (arrowheads) are visible in the animal (nuclear) region of the cloud. In a stage II oocyte (C) and an early stage III oocyte (D) the mitochondrial cloud is visible as an unfragmented, single entity with a layer of geminal granules at the vegetal cortex. (E) A Late stage III oocyte with a single layer of geminal granules (arrows) anchored at the vegetal cortex. Abbreviations: $\mathrm{mc}$, mitochondrial cloud; on, oocyte nucleus. Bars, $50 \mu \mathrm{m}$.

(Fig. 2B). We also noticed that the mitochondrial cloud was not in direct contact with the oocyte nucleus but rather a thin layer of homogenous cytoplasm devoid of mitochondria and other organelles was present between the mitochondrial cloud and the nucleus (Fig. 3A). We also noticed that in stage I oocytes mitochondrial cloud was positioned far from the vegetal cortex (Fig. $2 \mathrm{~A}$ ), but in early stage II oocytes it extended between the homogenous layer of cytoplasm surrounding the oocyte nucleus and the vegetal cortex, with its vegetal tip containing the layer of germinal granules apposing the vegetal cortex (Fig. 3A). Intriguingly, although we analyzed hundreds of sections of oocytes at devel-

Fig.3. Semithin sections through oocytes hybridized with Xcat2 $(A, C)$ and $X$ pat $(B)$ antisense $R N A$ probes, labeled with nanogold and silver enhanced. (A). In a stage II oocyte, the unfragmented mitochondrial cloud (mc) extends between the layer of homogenous cytoplasm (stars) surrounding the nucleus (on) and vegetal cortex. The germinal granules containing localized Xcat2 mRNA (artificially colored in red) are visible as a layer at the vegetal cortex in the METRO region. (B) Fragment of stage II oocyte showing the unfragmented mitochondrial cloud with a layer of Xpat mRNA (outlined artificially in red) at the vegetal cortex in the METRO region. (C) In a late stage III oocyte, germ plasm islands (fragments of mitochondrial cloud) are at the vegetal cortex. Arrows indicate the position of several of germ plasm islands, which are barely visible at such a low magnification. Bars in A,B, $25 \mu \mathrm{m}$; bar in $C, 50 \mu \mathrm{m}$. opmental stages I to III, we only extremely rarely observed germinal granules in the middle of the oocyte which should be the case if they were migrating within the fragments of the mitochondrial cloud. This observation prompted us to perform three-dimensional reconstruction of stage II oocytes, hybridized with Xcat2 and Xpat antisense RNA probes, to mark the positions of the germinal granules and the METRO region within the mitochondrial cloud and within the oocyte as a whole. These reconstructions showed clearly that the mitochondrial cloud in stage II oocytes was a continuous entity extending from the homogenous layer of cytoplasm surrounding the nucleus to the vegetal cortex, with the METRO-localized RNAs and all germinal granules located at its vegetal tip (Figs. 3,4). These three-dimensional reconstructions also showed the surface of the mitochondrial cloud to be uneven and convoluted at that stage, which probably explains why incidental sections through the cloud (Fig. 1B) showed islands within the cloud as opposed to a continuous unbroken structure. These findings indicate that METRO-localized RNAs and germinal granules reached the vegetal cortex of the oocyte before the onset of mitochondrial cloud fragmentation. We propose that after the initial aggregation (by some unknown mechanism) of germinal granules at the tip of the mitochondrial cloud in stage I oocytes, directional stretching of the mitochondrial cloud pushes the germinal granules and localized RNAs away from the nucleus and toward the vegetal cortex. Therefore germinal granules and METRO-localized RNAs do not translocate within the fragments of the mitochondrial cloud but rather are driven to the cortex through the expansion of the cloud. Thus the fragmentation of the cloud that occurs later in oogenesis is irrelevant to the movement of METRO-localized RNAs and germinal granules. Our revised model of the METRO pathway is depicted in Fig. 5. Although the mechanism by which the mitochondrial cloud expands remains unknown, one plausible cause is an increase in the abundance of membranous vesicles and in the number and size of mitochondria within cloud itself (Marinos and Billett, 1981).
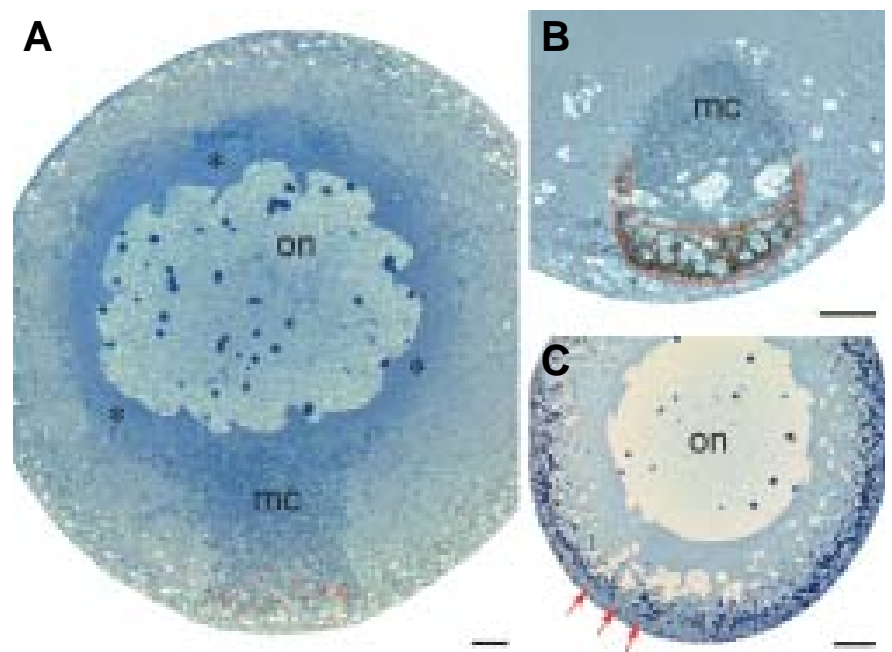

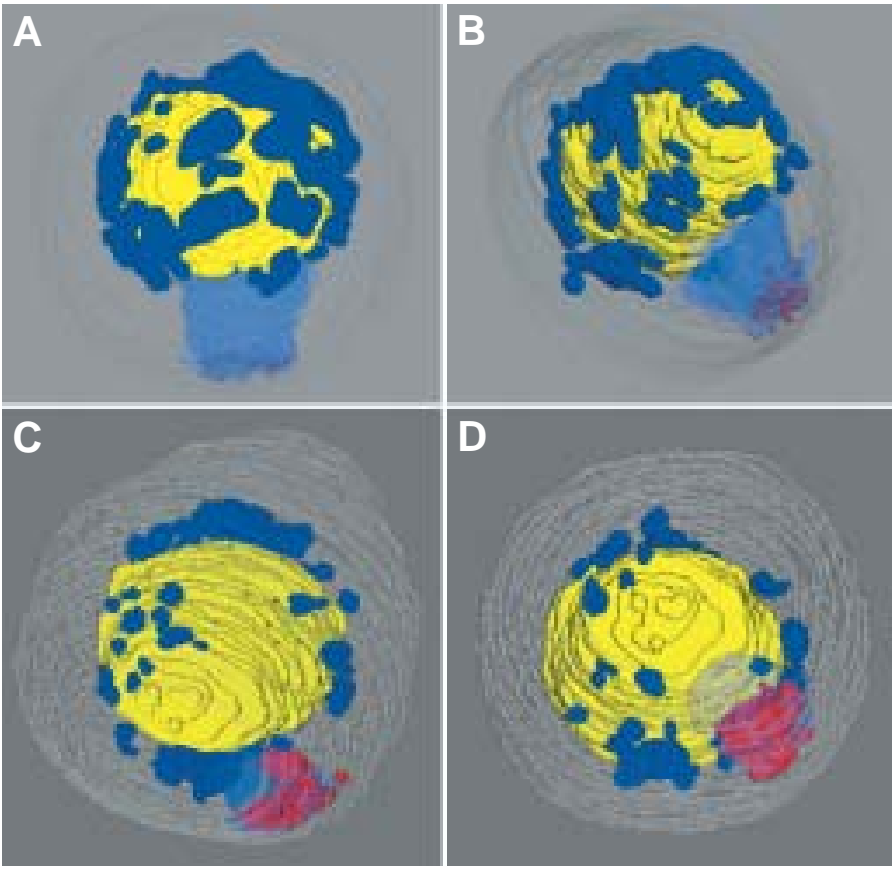

Fig. 4. Three-dimensional reconstructions of stage II oocytes hybridized with Xcat2 (A,B) or Xpat (C,D) mRNA probes, labeled with nanogold and silver enhanced. The mitochondrial cloud (light blue) is visible as an unfragmented entity extending between the nucleus (yellow) and vegetal cortex. The surface of the cloud is uneven and convoluted. Groups of mitochondria (secondary clouds, dark blue) are also visible around the nucleus. (A, B) Germinal granules (red dots) are located at the vegetal tip of the cloud at the METRO region of the oocyte. (C,D) Xpat MRNA (red) is concentrated in the METRO region of the cloud.

Another unresolved issue is the mechanism(s) responsible for the directionality of the mitochondrial cloud expansion. Because the dimensions of the homogenous layer of the cytoplasm separating the oocyte nucleus from the mitochondrial cloud does not increase from stage I oogenesis onward, it seems very unlikely that the process of pushing of mitochondrial cloud away from the

Fig. 5. Revised model of delivery of germinal granules and METROlocalized RNAs to the vegetal cortex of Xenopus oocytes. Steps 1 and 2 indicate stage I of oogenesis, in which the spherical mitochondrial cloud (light purple) is located near the nucleus (blue) but is separated from it by athin layer of homogenous cytoplasm (darkyellow). The germinal granules (dark purple) and METRO-localized RNAs are initially distributed at random within the cloud and subsequently become aggregated at its vegetal tip (the METRO region, step 2). In the old model (shown to the left), from stage Il of oogenesis onward, the mitochondrial cloud breaks down and the germinal granules and METRO-localized RNAs move within the mitochondrial cloud fragments (steps 3a and 4a) and become anchored at the vegetal cortex between stages III and VI of oogenesis (step 5). In the revised model (shown to the right), in stage II of oogenesis, the unbroken mitochondrial cloud expands to span between the nucleus and vegetal cortex, pushing the germinal granules and METRO-localized RNAs toward the vegetal cortex (steps $3 b$ and $4 b$ ) where they become anchored. In stage II of oogenesis the mitochondrial cloud become uneven and convoluted, which on the sections can give the impression of separated islands (step 4b). Subsequently, between stage III and VI of oogenesis, the mitochondrial cloud breaks down (step 4b'). The occasional "delayed" germinal granules are rarely visible in the fragments of the cloud (step $4 b^{\prime}$ ). nucleus is involved. Another process that needs to be studied is the mechanism by which germinal granules and localized RNAs segregate within the mitochondrial cloud in stage I oocytes.

\section{Materials and Methods}

\section{Animals}

Wild type Xenopus laevis oocytes were used in these studies as described in Kloc and Etkin (1999).

\section{Whole mount in situ hybridization}

The Xcat2 and Xpat plasmids and antisense RNA probes have been described elsewhere (Mosquera et al., 1993; Kloc and Etkin, 1995a, Hudson and Woodland, 1998). Probes used for in situ hybridization were antisense digoxigenin-labeled RNAs that were synthesized and hydrolyzed for $30 \mathrm{~min}$ as described in Kloc and Etkin (1999). All hybridization and post-hybridization steps, embedding and sectioning were performed as described in Kloc and Etkin, (1999) and Kloc et al. (2001). For light microscopy, hybridized oocytes were incubated with anti-digoxigenin antibody conjugated with alkaline phosphatase and stained in NBT/BCIP substrate (Roche). For electron microscopy oocytes were incubated with an anti-digoxigenin antibody conjugated with $0.8 \mathrm{~nm}$ gold (Roche) and treated with a silver enhancement kit (Ted Pella, Redding, CA) according to manufacturer's protocol. After the hybridization, oocytes were either embedded in Paraplast (for light microscopy) or in Epon (for electron microscopy) and sectioned as described previously (Kloc and Etkin, 1999 and Kloc et al. 2001.)

\section{Three-dimensional reconstruction}

Some of the material embedded for electron microscopy was serially sectioned for the three-dimensional reconstruction. Between 35 and 65 semi-thin $(0.7 \mu \mathrm{m})$ sections stained with methylene blue in $1 \%$ borax were used for each reconstruction. Three-dimensional reconstruction was performed as described in Kloc et al. (2002). In short, scanned photomicrographs of oocytes were processed on an SGI Onyx2 system (Silicon Graphics, Inc. Mountain View, CA) equipped with NAG (Numerical Algo-

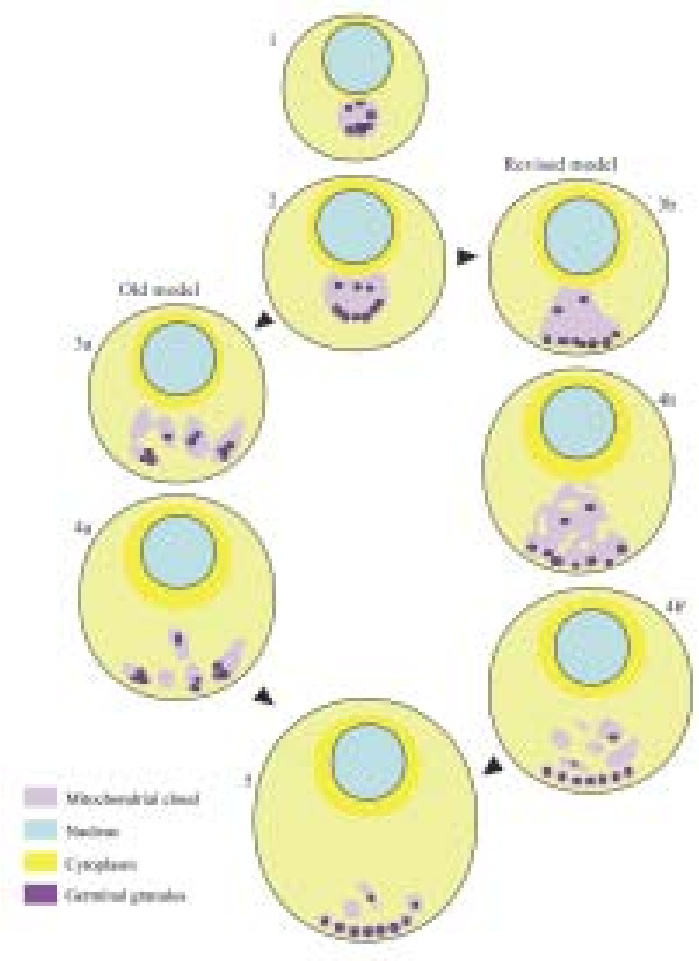


rithms Group, Oxford) Iris Explorer scientific visualization software. A typical data set comprising a cube of $300 \times 350 \times 8$ was interpolated to 64 $x 64 \times 64$. The resulting cube was further processed using the ImageVision modules within the Iris Explorer in addition to several customized software modules developed by Matthew T. Dougherty (Baylor College of Medicine) and VoxBlast 2.1 software (VayTek, Inc., Fairfield, lowa).

\section{Acknowledgements}

This work was supported by grants from NSF (to L. D. Etkin), The Polish State Committee for Scientific Research-KBN grant BW/4/IZ/2002 (to K.W), Core Grant CA16672 and NIH grant P41RR02250 (to M. T.D). We also thank Mr. Kenneth Dunner Jr. for electron microscopy work and Ms. Elzbieta Kisiel for superb work on figures.

\section{References}

AL-MUKHTAR, K A. K. and WEBB, A.C. (1971). An ultrastructural study of primordial germ cells, oogonia and early oocytes in Xenopus laevis. J. Embryol. Exp. Morphol. 26: 195-217.

BILLETT, F. S. and ADAM, E. (1976). The structure of the mitochondrial cloud of Xenopus laevis oocytes. J. Embryol. Exp. Morphol. 36: 697-710.

COGGINS, L. W. (1973). An ultrastructural and radioautographic study of early oogenesis in the toad Xenopus laevis. J. Cell Sci. 12: 71-93.

CZOLOWSKA, R. (1969). Observations on the origin of the 'germinal cytoplasm' in Xenopus laevis. J. Embryol. Exp. Morphol. 22: 229-251.

DE SMEDT, V., SZÖLLOLÖSI, D. and KLOC, M. (2000). The Balbiani body: asymmetry in the mammalian oocyte. Genesis 26: 208-212.

EDDY, E.M. (1975). Germ plasm and the differentiation of the germ cell line. Int. Rev. Cytol. 43, 229-280.

FORRISTALL, C., PONDEL. M., CHEN, L. and KING, M.L. (1995). Patterns of localization and cytoskeletal association of two vegetally localized RNAs, $\mathrm{Vg} 1$ and Xcat-2. Development. 121: 201-208.

GURAYA, S.S (1979). Recent advances in the morphology, cytochemistry and function of Balbiani's vitelline body in animal oocytes. Int. Rev. Cytol. 59:249-321.

HAUSEN, P. and RIEBESELL, M. (1991). The early development of Xenopus laevis. An atlas of the histology. Springer-Verlag Berlin, Heidelberg, New York.

HEASMAN, J., QUARMBY, J. and WYLIE, C. C. (1984). The mitochondrial cloud of Xenopus oocytes: the source of germinal granule material. Dev. Biol. 105: 458-69.

HOUSTON, D.W. and KING, M.L. (2000). Germ plasm and molecular determinants of germ cell fate. Curr. Top. Dev. Biol. 50: 155-181.

HUDSON, C. and WOODLAND, H. R. (1998). Xpat, a gene expressed specifically in germ plasm and primordial germ cells of Xenopus laevis. Mech. Dev. 73: 159-68.
KING, M.L. (1995). m RNA localization during frog oogenesis. In Localized RNAs. pp.137-148. Ed. Lipshitz H.D. Austin TX. R. G. Landes Company of Biomedical Publishers / Springer-Verlag.

KLOC, M. and ETKIN, L.D. (1995a). Two distinct pathways for the localization of RNAs at the vegetal cortex in Xenopus oocytes. Development 121: 287-297.

KLOC, M. and ETKIN, L. D. (1995b). Genetic pathways involved in the localization of RNA in Xenopus oocytes. pp-149-156. In Localized RNAs. Ed. Lipshitz, H. D. Austin Tx. R.G. Landes Company of Biomedical Publishers / Springer-Verlag.

KLOC, M., LARABELL, C., CHAN, A. P.Y. and ETKIN, L.D. (1998). Contribution of METRO pathway localized molecules to the organization of the germ cell lineage. Mech. Dev. 75: 81-93.

KLOC, M. and ETKIN,L.D. (1999). Analysis of localized RNAs in Xenopus oocytes. In A comparative methods approach to the study of oocytes and embryos. J. D. Richter, Ed., Oxford University Press, pp. 256-278.

KLOC, M., BILINSKI, S., CHAN, A.P.Y. and ETKIN, L. D. (2000). The targeting of Xcat2 mRNA to the germinal granules depends on cis-acting germinal granule localization element within the 3'UTR. Dev. Biol. 217: 221-229.

KLOC, M., BILINSKI,., CHAN, A. P.Y., ALLEN, L.H., ZEARFOSS, N.R. and ETKIN, L.D. (2001). RNA localization and germ cell determination in Xenopus. Int. Rev. Cytology 203: 63-91

KLOC, M., DOUGHERTY, M. T., BILINSKI, S., CHAN A.P., BREY, E., KING, M.L., PATRICK, C.W. and ETKIN, L.D. (2002). Three-dimensional ultrastructural analysis of RNA distribution within germinal granules of Xenopus. Dev. Biol. 241: 79-93

KLOC, M., BILINSKI, S. and ETKIN, L.D. (2004a). The Balbiani body and germ cell determinants:150 years later. Curr. Top. Dev. Biol. 59: 1-36.

KLOC, M., BILINSKI, S., DOUGHERTY, M.T., BREY, E. M. and ETKIN, L.D. (2004b). Formation, architecture and polarity of female germline cyst in Xenopus. Dev. Biol. 266: 43-61.

MARINOS, E. and BILLETT, F.S. (1981). Mitochondrial number, cytochrome oxidase and succinic dehydrogenase activity in Xenopus laevis oocytes. J. Embryol.Exp. Morphol. 62: 395-409.

MOSQUERA, L., FORRISTALL, C., ZHOU, Y. and KING, M. L. (1993). A RNA localized to the vegetal cortex of Xenopus oocytes encodes a protein with a nanoslike zinc finger domain. Development 117: 377-387

WYLIE, C. C., HOLWILL, S., O'DRISCOLL, M., SNAPE, A. and HEASMAN, J. (1985). Germ plasm and germ cell determination in Xenopus laevis as studied by cell transplantation analysis. Cold Spring Harb. Symp. Quant. Biol. 50: 37-43.

Received: September 2004

Reviewed by R eferees: December 2004 M odified by Authors and Accepted for Publication: January 2005 